\title{
Real-time diamagnetic flux measurements on ASDEX Upgrade
}

\author{
L.Giannone, ${ }^{1, \text { a) }}$ B.Geiger, ${ }^{1}$ R.Bilato, ${ }^{1}$ M.Maraschek, ${ }^{1}$ T.Odstrčil, ${ }^{1}$ R.Fischer, ${ }^{1}$ \\ J.C.Fuchs, ${ }^{1}$ P. J.McCarthy, ${ }^{2}$ V.Mertens, ${ }^{1}$ and K.H.Schuhbeck ${ }^{1}$ \\ 1) Max Planck Institute for Plasma Physics, 85748 Garching, \\ Germany \\ ${ }^{2)}$ Department of Physics, University College Cork, Cork T12 YN60, \\ Ireland
}

Real-time diamagnetic flux measurements are now available on ASDEX Upgrade. In contrast to the majority of diamagnetic flux measurements on other tokamaks, no analog summation of signals is necessary for measuring the change in toroidal flux or for removing contributions arising from unwanted coupling to the plasma and poloidal field coil currents. To achieve the highest possible sensitivity, the diamagnetic measurement and compensation coil integrators are triggered shortly before plasma initiation when the toroidal field coil current is close to its maximum. In this way the integration time can be chosen to measure only the small changes in flux due to the presence of plasma. Two identical plasma discharges with positive and negative magnetic field have shown that the alignment error with respect to the plasma current is negligible. The measured diamagnetic flux is compared to that predicted by TRANSP simulations. The poloidal beta inferred from the diamagnetic flux measurement is compared to the values calculated from magnetic equilibrium reconstruction codes. The diamagnetic flux measurement and TRANSP simulation can be used together to estimate the coupled power in discharges with dominant ion cyclotron resonance heating.

a) Louis.Giannone@ipp.mpg.de 


\section{INTRODUCTION}

The diamagnetic flux is the small difference in total toroidal flux with plasma and without plasma $^{1}$. A number of tokamaks have demonstrated the measurement of the diamagnetic

flux in real-time using analog summation circuits $^{2-5}$. In the superconducting tokamaks, KSTAR and Tore Supra, where the toroidal field is constant before and after the discharge, it is possible to carry out a diamagnetic flux loop measurement without using an analog summation circuit ${ }^{6,7}$. In the W7-AS stellarator, the diamagnetic flux loop integrator was initiated in the flat top of the toroidal field current and this makes digital compensation of the signal possible ${ }^{8}$. In DIII-D, the diamagnetic flux loop integrators are separately turned on both before the start of toroidal field current and in the flat top for comparison ${ }^{5}$. From the diamagnetic flux measurement, the value of diamagnetic plasma energy and thus the poloidal beta can be calculated ${ }^{9}$. The diamagnetic flux loop measurement is also desirable for tokamak equilibrium reconstruction as it can provide additional information on the central value of the safety factor ${ }^{10}$.

In Section II, the experimental basis for real-time diamagnetic flux measurements in ASDEX Upgrade is presented and the calibration of the relative sensitivities of the diamagnetic measurement and compensation loop using vacuum discharges with toroidal field coil currents only are considered. The theoretical background of the diamagnetic flux measurement is summarised in section III. A small misalignment of the diamagnetic loops with respect to the toroidal direction means that unwanted signals are generated by the poloidal field coil currents and the plasma current. The procedure to determine the mutual inductance of the diamagnetic loops and the poloidal field coils to calculate the necessary corrections in real-time is discussed in Section IV. The experiments performed to evaluate the contribution of plasma current to the diamagnetic flux signals is also discussed in Section IV. Simulations using TRANSP are carried out to predict the diamagnetic flux and the results are compared to the measurements in Section V. In addition, values of poloidal beta derived from the diamagnetic flux loop measurement are compared to those obtained from magnetic equilibrium reconstruction codes. 


\section{DIAMAGNETIC FLUX LOOP}

A schematic diagram of the external diamagnetic loop on ASDEX Upgrade is shown

in Fig. 1. The measurement loop with area $\mathrm{S}_{\text {meas }} \approx 3.37 \mathrm{~m}^{2}$ and the compensation loop with area $\mathrm{S}_{\text {comp }} \approx 3.74 \mathrm{~m}^{2}$ are indicated. Two sets of internal and three sets of external diamagnetic loops are installed on ASDEX Upgrade ${ }^{11}$. The external measurement loop is 2 windings in the poloidal direction around the outside of the vacuum vessel. The compensation loop is used to measure the vacuum toroidal field and is made with coils aligned in a guide tube mounted in a $10 \mathrm{~mm}$ deep machined groove support structure on the outside of the vacuum vessel. The measurement loop is also wound on the same support structure and this ensures that the measurement and compensation loops cannot move in the toroidal magnetic field independently. Rogowski coils are mounted on each of the 16 toroidal field coils and the unintegrated signals are used by the control system to ensure that current is flowing in each toroidal field coil.

The internal measurement loop is a single turn mounted inside the vacuum vessel. A set of 12 internal magnetic probes distributed in poloidal angle was used to measure the toroidal component of the magnetic flux for the compensation of the toroidal flux of the internal measurement loop. Both the inner and outer diamagnetic loops are made of PEEK insulated copper wires (AWG 22) with $1.95 \mathrm{~mm}$ outside diameter inside a stainless steel guide tube of cross-section $8 \mathrm{~mm} \times 8 \mathrm{~mm}$ and thickness $1 \mathrm{~mm}$. The diamagnetic loops are not located in the vicinity of the leads to the toroidal and poloidal field coils, in order that stray field coupling is minimised ${ }^{2}$. The signal for the internal compensation flux is presently suffering from drifts during neutral beam ion (NBI) and ion cyclotron resonance (ICRH) heating, so they cannot be used for diamagnetic flux loop measurements.

The flux in the measurement loop, $\Phi_{m}$, and in the compensation loop, $\Phi_{c}$, are then given by the surface integral :

$$
\begin{aligned}
\Phi_{m} & =\int_{S_{\text {meas }}} B_{\phi} d S \\
\Phi_{c} & =\int_{S_{\text {comp }}} B_{\phi} d S
\end{aligned}
$$




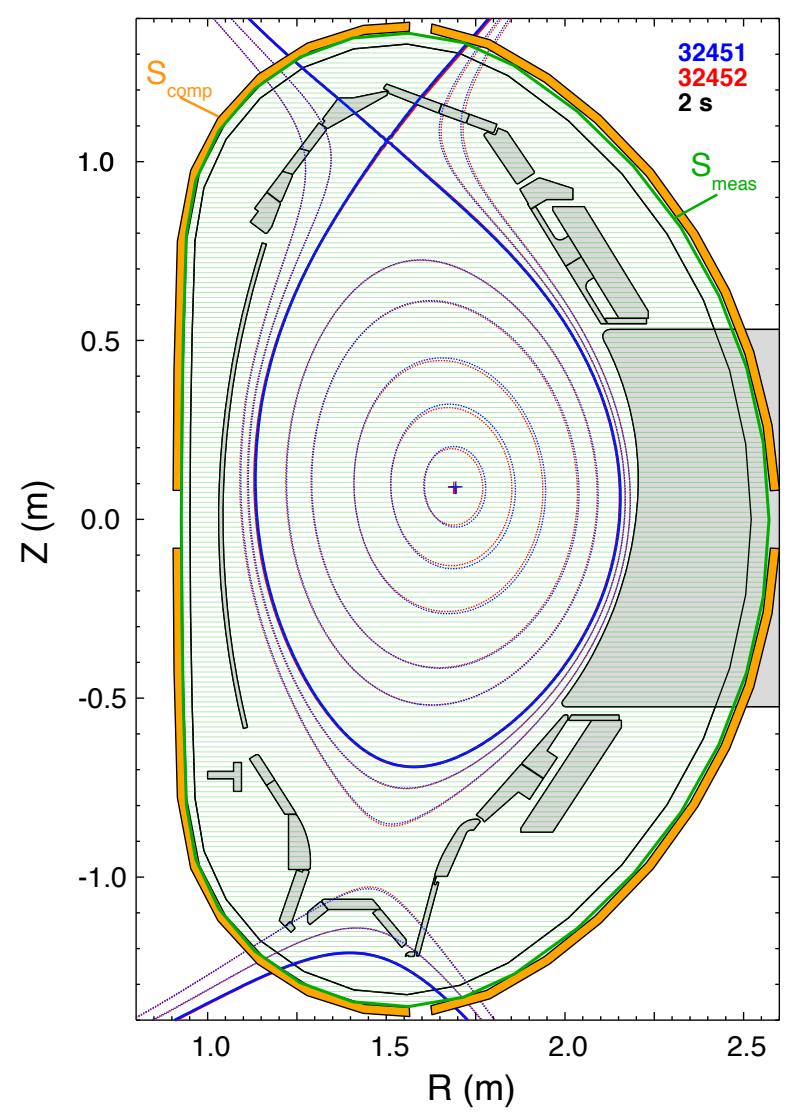

FIG. 1. Schematic diagram of the external diamagnetic loop on ASDEX Upgrade. The external measurement diamagnetic loop (green) is 2 turns mounted on a frame surrounding the vacuum vessel in the poloidal direction. The external compensation diamagnetic loop (yellow) is also mounted on this frame. The contours of the poloidal flux of the magnetic equilibrium for a discharge with negative (blue) and positive (red) toroidal field are also plotted.

The diamagnetic flux, $\Delta \Phi$, is then given by :

$$
\Delta \Phi=\Phi_{m}-\alpha_{C} \Phi_{c}
$$

Vacuum field measurements with a steady value of current in the toroidal field coil are used to establish the relative sensitivity of the measurement and compensation loops and determine the coefficient $\alpha_{C}$ necessary to have $\Delta \Phi=0$. The vacuum discharges for this purpose are now considered. The diamagnetic measurement and compensation loop flux signals for the external loops are shown in Fig. 2. The integrators of each measurement were triggered close to flat top phase of the toroidal field coil current. The scale factor of the diamagnetic compensation loop signal has been chosen to match the diamagnetic 
measurement loop signal. It is necessary to introduce a $20 \mathrm{~ms}$ delay in the compensation loop signal to allow for field diffusion into the vacuum vessel and the later response of the measurement loop signal. The diamagnetic flux is then simply calculated by digital subtraction of the delayed compensation loop flux signal from the measurement loop flux signal. The external diamagnetic flux is therefore close to zero in this calibration discharge as shown in the lower trace of Fig. 2. The drift and noise level of this difference is sufficiently small to provide a useful external diamagnetic flux signal for ASDEX Upgrade discharges.

The diamagnetic flux is typically $18 \mathrm{mWb}$ in a $1 \mathrm{MA}$ ohmic discharge with $2.5 \mathrm{~T}$ toroidal magnetic field on ASDEX Upgrade. The vacuum toroidal magnetic flux is therefore 470 times greater than the diamagnetic flux. As the diamagnetic flux to be measured in an auxiliary heated plasma can typically be up to a factor $10^{4}$ smaller than the toroidal flux, the difference of the measurement and compensation diamagnetic flux in earlier experiments has been produced in real-time by an electronic summation circuit $^{2-5}$. The compensation for poloidal field and ohmic heating coil currents was carried out by analog subtraction of an appropriate fraction of the measured coil current. In a tokamak with superconducting toroidal field coils, the toroidal field coil currents flow continuously before and after the discharge. Therefore, the change in toroidal flux during a discharge does not require an electronic summation circuit for the subtraction of a large change in toroidal flux. However, the pulse length of current in the copper toroidal field coils in ASDEX Upgrade limits the duration of the flat top magnetic field to $15 \mathrm{~s}$. To achieve the highest possible sensitivity, the diamagnetic loop measurement and compensation coil integrators are triggered shortly before plasma initiation when the toroidal field coil current is close to its maximum. In this way the integration time can be chosen to measure only the small changes in flux due to the presence of plasma. These integrators have an integration time constant of $20 \mathrm{~ms}$. The diamagnetic flux is then calculated in real-time by digital subtraction of the delayed compensation flux signal from the measurement flux signal. The diamagnetic flux must be also compensated in real-time for signals in the diamagnetic loops produced by currents flowing in the poloidal field and ohmic heating coils. This point is discussed in detail in section IV. 


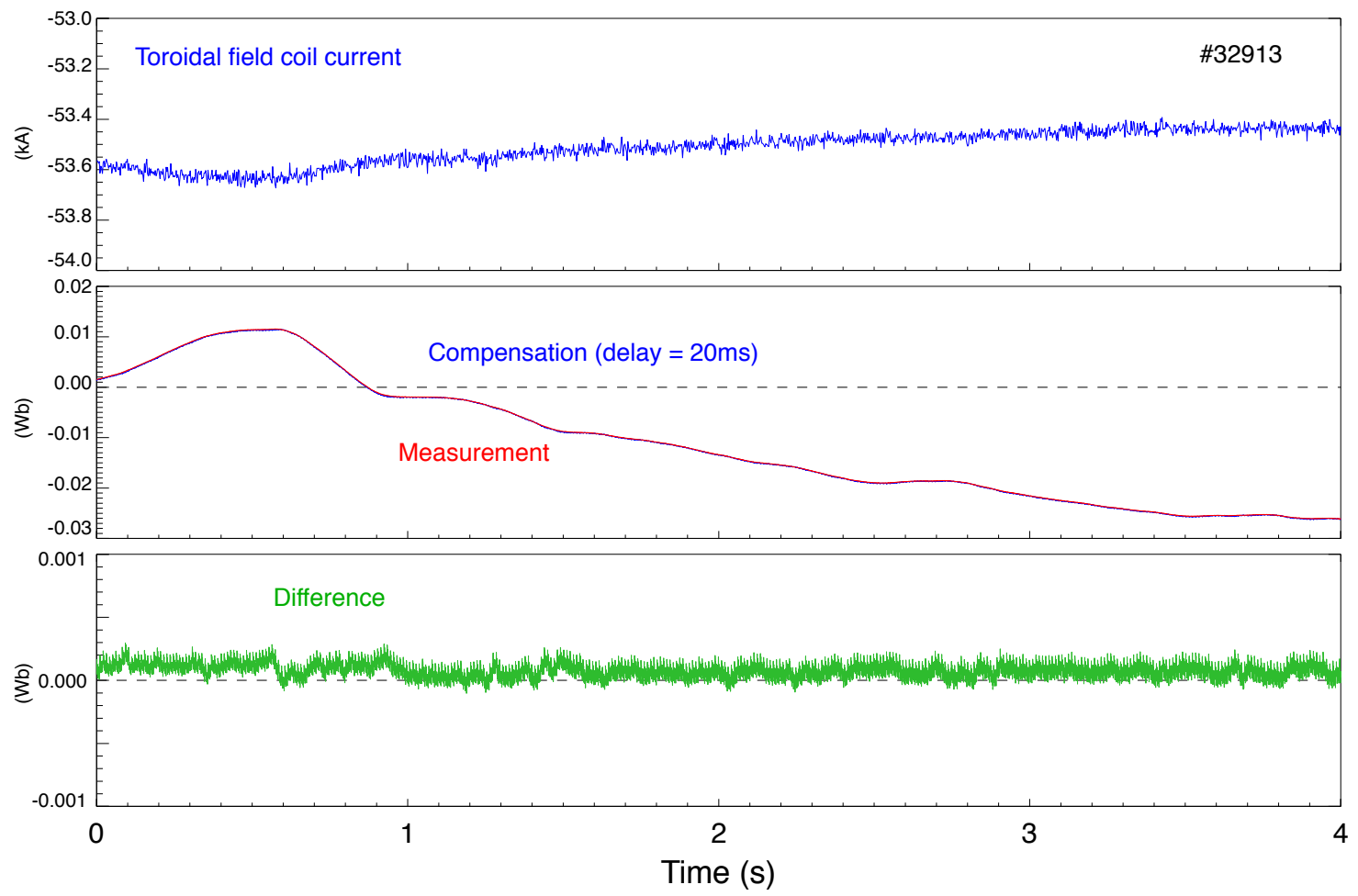

FIG. 2. The integrators are triggered close to flat top phase of the toroidal field coil current in a vacuum discharge. The external diamagnetic measurement (red) and compensation (blue) loop flux signals are shown. The diamagnetic flux is the difference (green) in flux measured by the external measurement and compensation coils and is close to zero. It is necessary to introduce a $20 \mathrm{~ms}$ delay in the compensation loop signal to allow for field diffusion into the vacuum vessel and the later response of the measurement loop signal.

\section{THEORY}

By considering the components of the force balance equation along the major radius and along the minor radius :

$$
\nabla p=\underline{j} \times \underline{B}=\frac{\nabla \times \underline{B}}{\mu_{o}} \times \underline{B}
$$

where $p$ is the plasma pressure of an isotropic plasma, $j$ is the plasma current density and $\underline{B}$ the magnetic field, two equations for volume integrals relating the plasma energy and internal inductance to surface integrals of the magnitude and direction of the magnetic field at the measuring surface and the diamagnetic flux can be derived ${ }^{1,9,12}$. The ratio of the plasma pressure to the pressure of the magnetic field associated with the plasma current or 
poloidal beta, $\beta_{p}$, is defined by :

$$
\beta_{p}=\frac{2 \mu_{o}}{V \bar{B}_{p a}^{2}} \int p d V=\frac{4 \pi s^{2}}{V \mu_{o} I_{p}^{2}} \int R p d R d Z
$$

and the internal inductance per unit length of the plasma column, $l_{i}$, is defined by :

$$
l_{i}=\frac{1}{V \bar{B}_{p a}^{2}} \int b_{p o l}^{2} d V=\frac{2 \pi s^{2}}{V\left(\mu_{o} I_{p}\right)^{2}} \int R b_{p o l}^{2} d R d Z
$$

where $V$ is the plasma volume, $\bar{B}_{p a}=\left(\mu_{o} I_{p}\right) / s$ is the mean poloidal field around the contour of the plasma boundary, and $s$ is the distance around the contour of the plasma boundary. Then $\beta_{p}$ and $l_{i}$ can be expressed in terms of the Shafranov integrals, $S_{1}$ and $S_{2}$, which are contour integrals on the last closed flux surface of a tokamak magnetic equilibrium ${ }^{9,13}$ :

$$
\begin{aligned}
S_{1} & =\frac{\pi}{V \bar{B}_{p a}^{2}} \iint R b_{p o l}^{2}\left(\left(R-R_{o}\right) \bar{e}_{R}+Z \bar{e}_{Z}\right) \cdot \bar{n} d s \\
& =\frac{\pi s^{2}}{V\left(\mu_{o} I_{p}\right)^{2}} \oint_{C} R b_{p o l}^{2}\left(\left(R-R_{o}\right) \bar{e}_{R}+Z \bar{e}_{Z}\right) \cdot \bar{n} d s \\
S_{2} & =\frac{\pi}{V \bar{B}_{p a}^{2}} \iint R b_{p o l}^{2} R_{o} \bar{e}_{R} \cdot \bar{n} d s \\
& =\frac{\pi s^{2}}{V\left(\mu_{o} I_{p}\right)^{2}} \oint_{C} R b_{p o l}^{2} R_{o} \bar{e}_{R} \cdot \bar{n} d s
\end{aligned}
$$

where $\bar{n}$ is the vector perpendicular to the line element of the contour, $b_{p o l}$ is the poloidal magnetic field and $R_{o}$ is the major radius of the torus axis. Then $\beta_{p}$ and $l_{i}$ can be expressed in terms of these integrals :

$$
\begin{aligned}
\beta_{M H D}+l_{i} / 2 & =\left(S_{1}+S_{2}\left(1+R_{c} / R_{o}\right)\right) / 2 \\
\beta_{D I A}-\mu_{I} & =S_{1}+S_{2}\left(1-R_{c} / R_{o}\right)
\end{aligned}
$$

and the diamagnetic parameter, $\mu_{I}$, is proportional to the diamagnetic flux, $\Delta \Phi$ :

$$
\begin{gathered}
\mu_{I}=\frac{2 \pi R_{o}}{V} \frac{2 B_{\phi o}}{\bar{B}_{p a}^{2}} \Delta \Phi \\
\Delta \Phi=-\int\left(B_{\phi}-B_{\phi o}\right) d S
\end{gathered}
$$


where $B_{\phi}$ is the toroidal magnetic field in the presence of plasma, $B_{\phi o}$ is the toroidal magnetic field in the absence of plasma and $\mathrm{V} /\left(2 \pi \mathrm{R}_{o}\right)$ is the area of the plasma in the poloidal plane ${ }^{14}$. The values of $\beta_{M H D}$ and $\beta_{D I A}$ are the values of $\beta_{p}$ determined either from the reconstructed magnetic equilibrium or from the diamagnetic flux measurement. It should be noted that that the two independently determined values of poloidal beta do not have to agree unless the pressure is isotropic. $R_{c}$ is the radius of the plasma current centroid defined by :

$$
R_{c}^{2}=\frac{1}{\mu_{o} I_{p}} \oint_{C} R^{2} b_{p o l} d s
$$

It is useful to recall that the energy content of the plasma can be expressed in terms of $\beta_{p}{ }^{1}$ :

$$
W=\frac{3}{2} \int p d V=\frac{3}{2} \frac{V \mu_{o} I_{p}^{2}}{s^{2}} \beta_{p}
$$

To arrive at a simplified expression for $\beta_{D I A}$ in terms of $\Delta \Phi$, the small second term on the right hand side of Eqn. 9 is neglected and $S_{1} \approx 1$ is used. For an ellipse of minor radius a and major radius $b$, the area of the ellipse is approximately $\pi \mathrm{ab}$ and the circumference approximately by $\pi(\mathrm{a}+\mathrm{b})$. Using the approximation $(1+\kappa)^{2}=2\left(1+\kappa^{2}\right)$ where $\kappa=\mathrm{b} / \mathrm{a}$ is the plasma elongation, then equation 9 can be simplified to ${ }^{3,5,14}$ :

$$
\beta_{D I A}=1+\frac{\kappa^{2}+1}{2 \kappa} \frac{8 \pi B_{\phi o}}{\left(\mu_{o} I_{p}\right)^{2}} \Delta \Phi
$$

For $\beta_{D I A}<1$, the toroidal field magnitude is larger than the vacuum toroidal field magnitude. For $\beta_{D I A}>1$, the toroidal field magnitude is smaller than the vacuum toroidal field magnitude. The convention used for plotting the measured diamagnetic flux will reflect this physical effect even though the sign of the measurement changes when the toroidal field direction changes. The diamagnetic flux therefore provides a continuous measure of the poloidal beta without requiring density and temperature profiles of the ions or electrons. It has been noted ${ }^{14}$ that the poloidal beta, as determined from diamagnetic measurements using Eq. 14, is related to the perpendicular component of pressure, whereas the one determined from magnetic equilibrium measurements is related to the perpendicular, parallel and flow components of pressure. Thus, for shaped tokamaks, magnetic equilibrium measurements together with diamagnetic measurements may be used to determine the plasma 
anisotropy. The values of $\beta_{M H D}$ and $\beta_{D I A}$ were compared in Doublet III using the EFIT magnetic equilibrium reconstruction code ${ }^{15}$. In this study the two values agreed over a large number of discharges.

In an anisotropic plasma, the perpendicular pressure, $p_{\perp}$ and parallel pressure, $p_{\|}$are not equal. The perpendicular beta, $\beta_{p \perp}$ is then defined by ${ }^{14}$,

$$
\beta_{p \perp}=\frac{2 \mu_{o}}{V \bar{B}_{p a}^{2}} \int p_{\perp} d V
$$

Good examples of an anisotropic plasma are found in ICRH discharges on Tore Supra ${ }^{6}$. Because of the contribution of fast particles to the perpendicular energy, the diamagnetic energy is greater than the plasma energy from kinetic profiles. Similar ASDEX Upgrade discharges with ICRH are considered in section 5.

\section{COMPENSATION}

This section is devoted to compensating the diamagnetic loop signals generated by their mutual inductance to the poloidal field coils and plasma current resulting from a small misalignment. In the ideal case, the measurement and compensation coils of the diamagnetic loop are perfectly axisymmetric and the diamagnetic loops are exactly perpendicular to the

poloidal field coils and no magnetic flux should be measured when currents are flowing in the poloidal field coils.

For a test discharge with individual currents in each of the poloidal field coils, the coupled flux to the measurement and compensation coils of the external diamagnetic loop are recorded. The mutual inductance to each poloidal field coil can then be calculated and a matrix multiplication can be performed to apply the appropriate corrections during a plasma discharge. The measured diamagnetic flux for the external diamagnetic loop after correction is plotted in the lower frame of Fig. 3. In the flat top phase of the current pulses, the diamagnetic flux is well corrected and close to zero. The signal in the current ramp phase is due to the generation of vessel currents that also produce a signal in the measurement and compensation loop. Corrections for current ramps are not applied, as in the flat top phase of a discharge there are only slow changes in the poloidal field coil currents for shape control. The two poloidal field coils used for position control (ICoIo and ICoIu) do not 
generate significantly large signals in their current ramp up phase.

ASDEX Upgrade uses magnetic perturbation coils for error field correction and ELM control $^{16}$. In dedicated vacuum discharges with $n=1$ coil currents pulses at the maximum value of $1 \mathrm{kA}$, a pickup of $0.5 \mathrm{mWb}$ was measured. This pickup is not being corrected for at present in real-time. It is planned to introduce the necessary corrections for $\mathrm{n}=1$ and $\mathrm{n}>1$ perturbations of the diamagnetic flux in real-time in the future. This will be achieved in a similar manner as the correction of perturbations generated by the poloidal field coil currents.

In Fig. 4 the diamagnetic flux measurement for a discharge with positive and negative toroidal magnetic field is shown. The plasma remained in L-mode as only $400 \mathrm{~kW}$ of ECRH heating was used. The magnetic equilibrium for these two discharges is shown in Fig. 1. The plasma current and electron density in the flat top phase are controlled to be as identical as possible. The difference in diamagnetic flux is considered to be small enough to ignore the compensation of the diamagnetic loop signals for the plasma current.

\section{TRANSP SIMULATIONS}

The transport code, TRANSP ${ }^{17,18}$, allows the diamagnetic flux (parameter DFLUX) to be calculated and compared to the experimental measurements in ASDEX Upgrade. This code uses inputs, such as the measured kinetic plasma profiles, power and timing of heating sources. The code has its own equilibrium solver that considers the plasma pressure, the total plasma current and the position of the separatrix. The position of the separatrix is determined by the offline ASDEX Upgrade magnetic equilibrium reconstruction code, CLISTE $^{19}$. However, in contrast to CLISTE, TRANSP simulates the fast-ion distribution function which has an impact on the diamagnetic flux. Therefore TRANSP is the appropriate tool for the following comparisons.

In Fig. 5, the measured diamagnetic flux is compared to that predicted by the TRANSP simulation for a discharge with neutral beam ion heating (NBI) and ion cyclotron resonance heating $(\mathrm{ICRH})$. In Fig. 6, the measured diamagnetic flux is compared to that predicted by the TRANSP simulation for a discharge with neutral beam and electron cyclotron heating. The poloidal beta inferred from the diamagnetic flux measurement ${ }^{19}, \beta_{D I A}$, is in good agree-

ment with the values calculated from the real-time ${ }^{20,21}$ and offline magnetic equilibrium re- 
32855

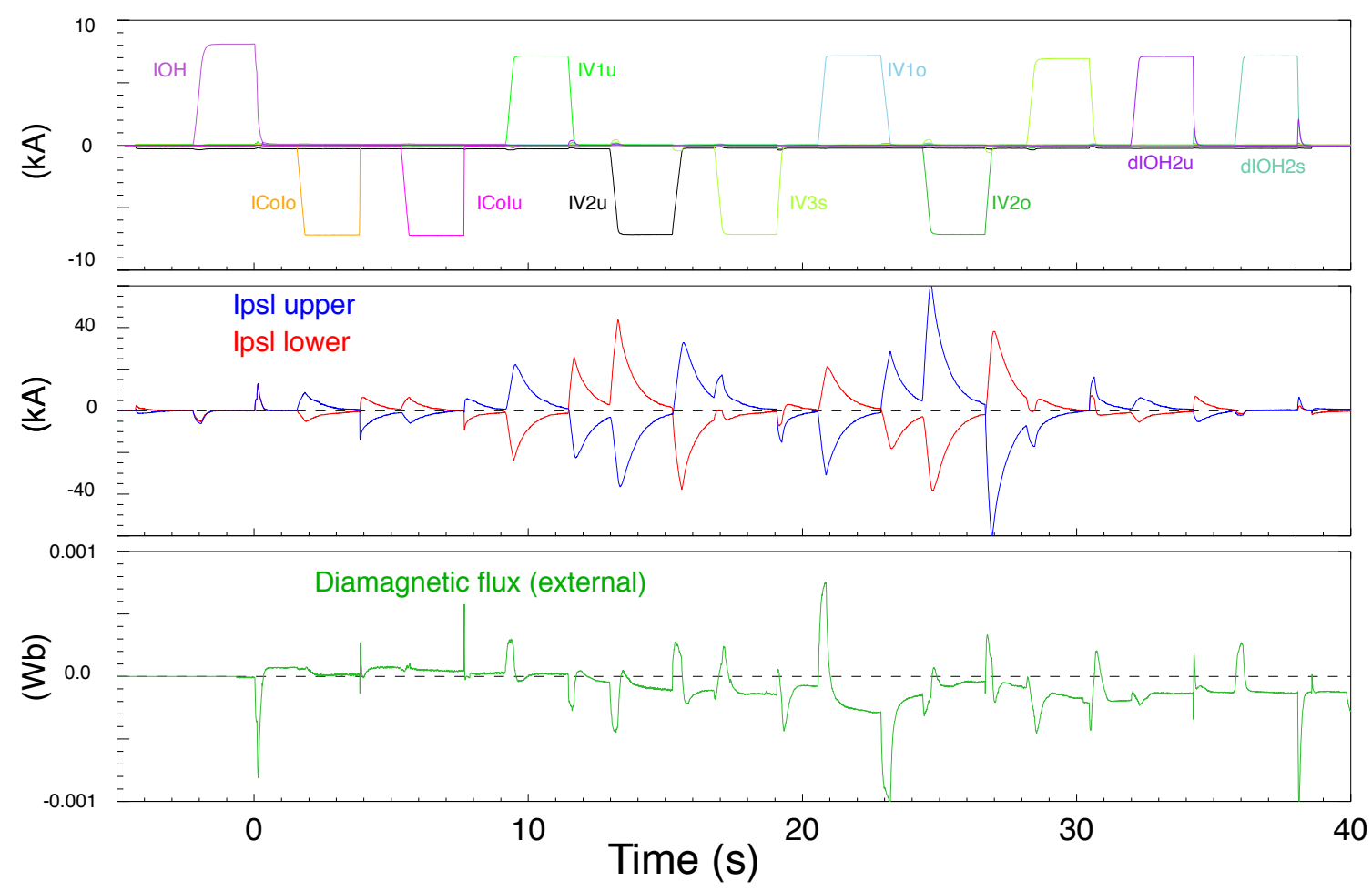

FIG. 3. The currents in the poloidal field and ohmic heating coils are shown the upper frame and the currents in the lower (red) and upper (blue) passive stabilising loop in the second frame. The diamagnetic flux measurement for the external diamagnetic loop (green), corrected for the mutual inductance coupling due to misalignment to the poloidal field and ohmic transformer coils are shown in the lower frame.

construction codes, $\beta_{M H D}$, in the steady state phase of the discharges. In the discharge with electron cyclotron heating, the increase in poloidal beta with time is due to a steady increase in the nitrogen seeding introduced for reduction of the heat load to the divertor target ${ }^{22}$. The details of implementation of the real-time equilibrium code, JANET, and the differences between the real-time and offline equilibrium code, CLISTE, have been discussed ${ }^{20}$.

It can be seen in the early phases of the discharges in Fig. 5 and Fig. 6 that the difference between $\beta_{D I A}$ and $\beta_{M H D}$ increased with decreasing $\beta_{M H D}$. In the real-time equilibrium reconstruction code this discrepancy could be reduced in low $\beta_{M H D}$ discharges by adjusting the value of the constraint for $\mathrm{q}(0)$. A detailed comparison of $W_{M H D}$ and $W_{D I A}$ in JET has also shown a systematic difference at low $\beta_{p}{ }^{23}$. At low $\beta_{p}, \mathrm{~W}_{M H D}$ could be increased by $0.5 \mathrm{MJ}$ when the measured diamagnetic flux was used as a constraint. It has been 

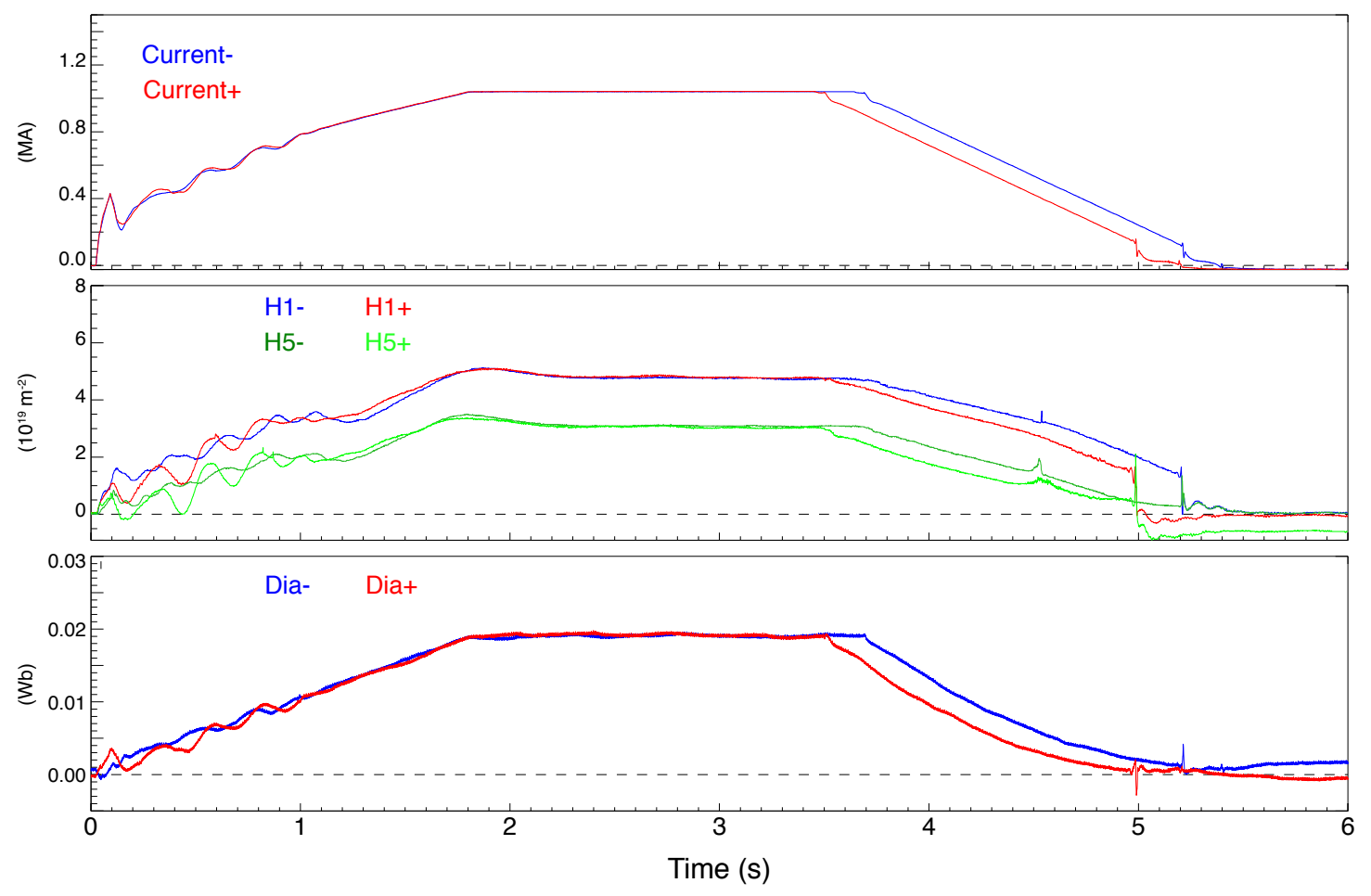

FIG. 4. Comparing two discharges with negative (blue) and positive (red) toroidal field shows that the coupling of the diamagnetic loop flux measurement to plasma current is negligible. The plasma current and the central and edge electron density line integrals of the DCN laser ( H1 and H5) are controlled to be as equal as possible. The diamagnetic flux for negative toroidal field has been inverted to illustrate the lack of dependence of the diamagnetic flux on magnetic field direction.

noted that it is also necessary to use the measured diamagnetic flux as a constraint for the magnetic equilibrium reconstruction for nearly circular plasmas, as the separation $\beta_{p}$ and $l_{i}$ is only possible in an elongated tokamak magnetic equilibrium ${ }^{15}$. It is claimed that magnetic equilibrium reconstruction constrained by the measured diamagnetic flux could provide an estimate of the central value of the safety factor ${ }^{10}$.

In Fig. 7, the measured diamagnetic flux is shown for a discharge with dominant ion cyclotron resonance heating (ICRH). The diamagnetic flux predicted by TRANSP is plotted for 4 different fractions of coupled ICRH power. The power reflected by the ICRH antenna and line losses mean that only part of the nominal generator power is coupled to the plasma. Best agreement between the measured and predicted diamagnetic flux is found when only half the ICRH generator power is coupled into the plasma. This result suggests that a 

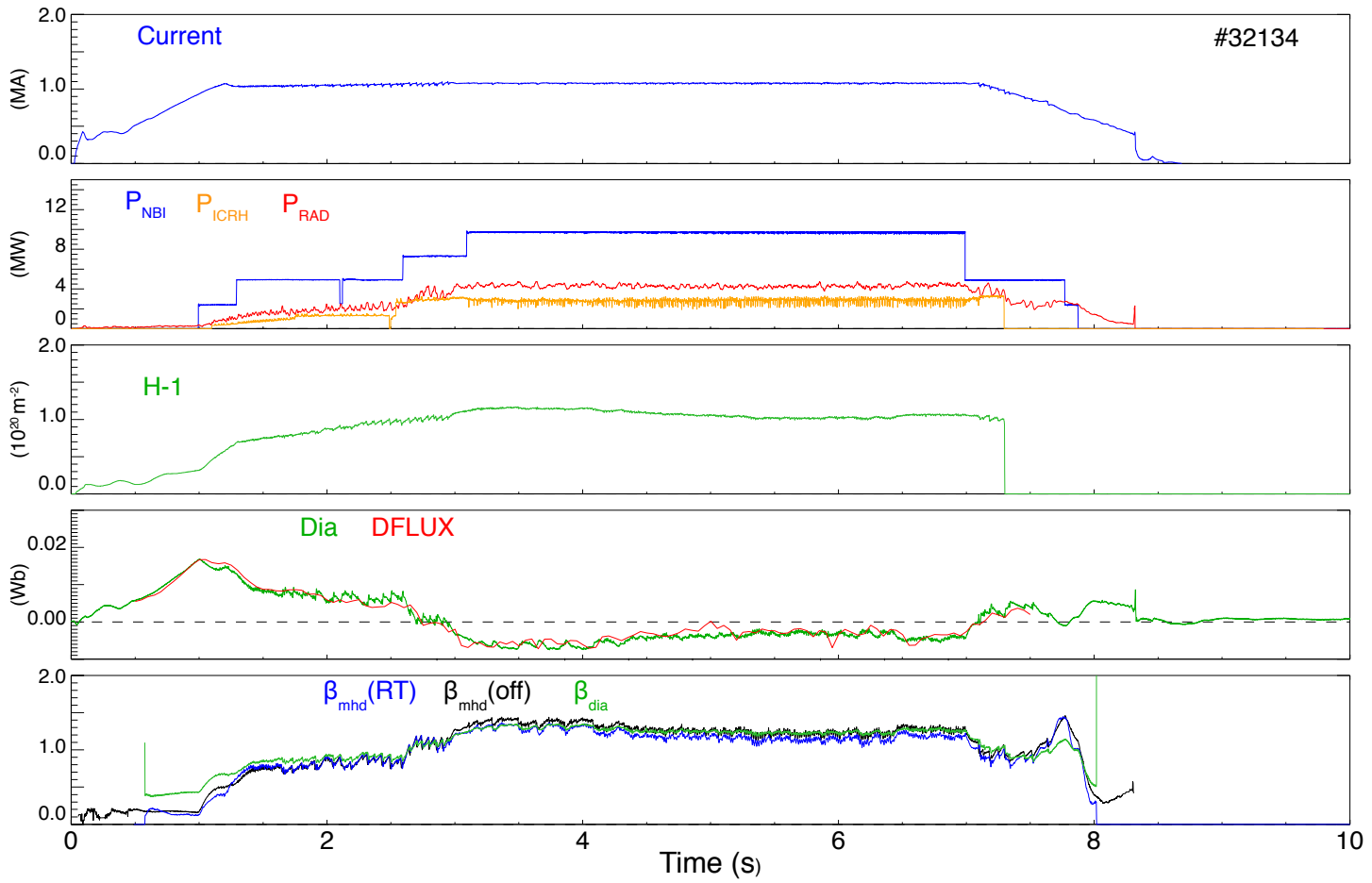

FIG. 5. Comparison of the diamagnetic flux and beta poloidal with the TRANSP simulation in a discharge with NBI and ICRH heating. The time evolution of the plasma current and the central electron density line integral of the DCN laser ( H1) are shown. The measured diamagnetic flux, Dia (green), and predicted values from TRANSP, DFLUX (red), are in good agreement. The poloidal beta inferred from the measured diamagnetic flux, $\beta_{D I A}$ (green), is in good agreement in the steady state phase of the discharge with the poloidal beta calculated from the real-time and offline magnetic equilibrium ( $\beta_{M H D}(R T)$ (blue) and $\beta_{M H D}($ off $)$ (black)).

diamagnetic flux measurement and TRANSP simulation can be used together to estimate the coupled power in ICRH dominated discharges. The values of $\beta_{M H D}$ calculated from the magnetic equilibrium reconstruction codes are lower than $\beta_{D I A}$ inferred from the measured diamagnetic flux. This feature is expected for an anisotropic plasma, for example when the perpendicular pressure is greater than the parallel pressure.

\section{CONCLUSION}

Real-time diamagnetic flux measurements for ASDEX Upgrade are now in routine operation. The measurement and compensation coil integrators are initiated close to the flat top phase of the toroidal magnetic field prior to the start of the plasma current ramp. The cal- 


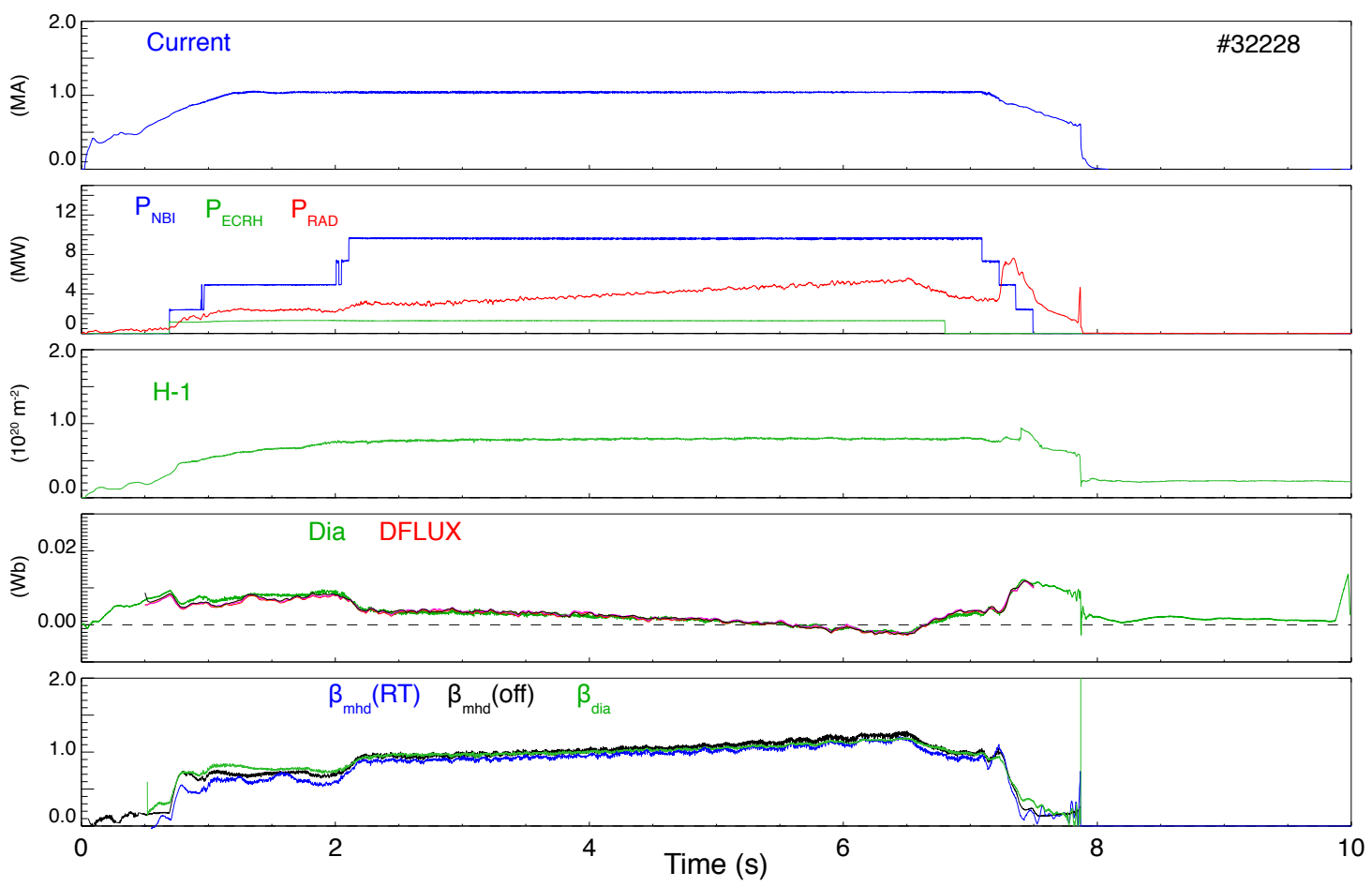

FIG. 6. Comparison of the diamagnetic flux and beta poloidal with the TRANSP simulation in a discharge with NBI and ECRH heating. The time evolution of the plasma current and the central electron density line integral of the DCN laser ( H1) are shown. The measured diamagnetic flux, Dia (green), and predicted values from TRANSP, DFLUX (red), are in good agreement. The poloidal beta inferred from the measured diamagnetic flux, $\beta_{D I A}$ (green), is in good agreement in the steady state phase of the discharge with the poloidal beta calculated from the real-time and offline magnetic equilibrium ( $\beta_{M H D}(R T)$ (blue) and $\beta_{M H D}($ off $)$ (black) ).

ibration of the relative magnitude of internal and external measurement and compensation loop signals are determined in dedicated discharges with toroidal field only. The mutual inductance of the diamagnetic loop with the poloidal field and ohmic heating coils are found by measuring the response to currents in each coil. The measured diamagnetic flux and the prediction by TRANSP for discharges with a variety of heating schemes are found to be in good agreement. In the steady state phase of these discharges, good agreement is found between the values for $\beta_{D I A}$ inferred from the diamagnetic flux and $\beta_{M H D}$ from magnetic reconstruction codes. In the early phases of these discharges, the difference in $\beta_{D I A}$ and $\beta_{M H D}$ increased with decreasing $\beta_{M H D}$. To improve agreement, the diamagnetic flux will be used as an additional magnetic equilibrium reconstruction constraint. In a discharge with dominant ICRH power, the best agreement between the measured diamagnetic flux and that 

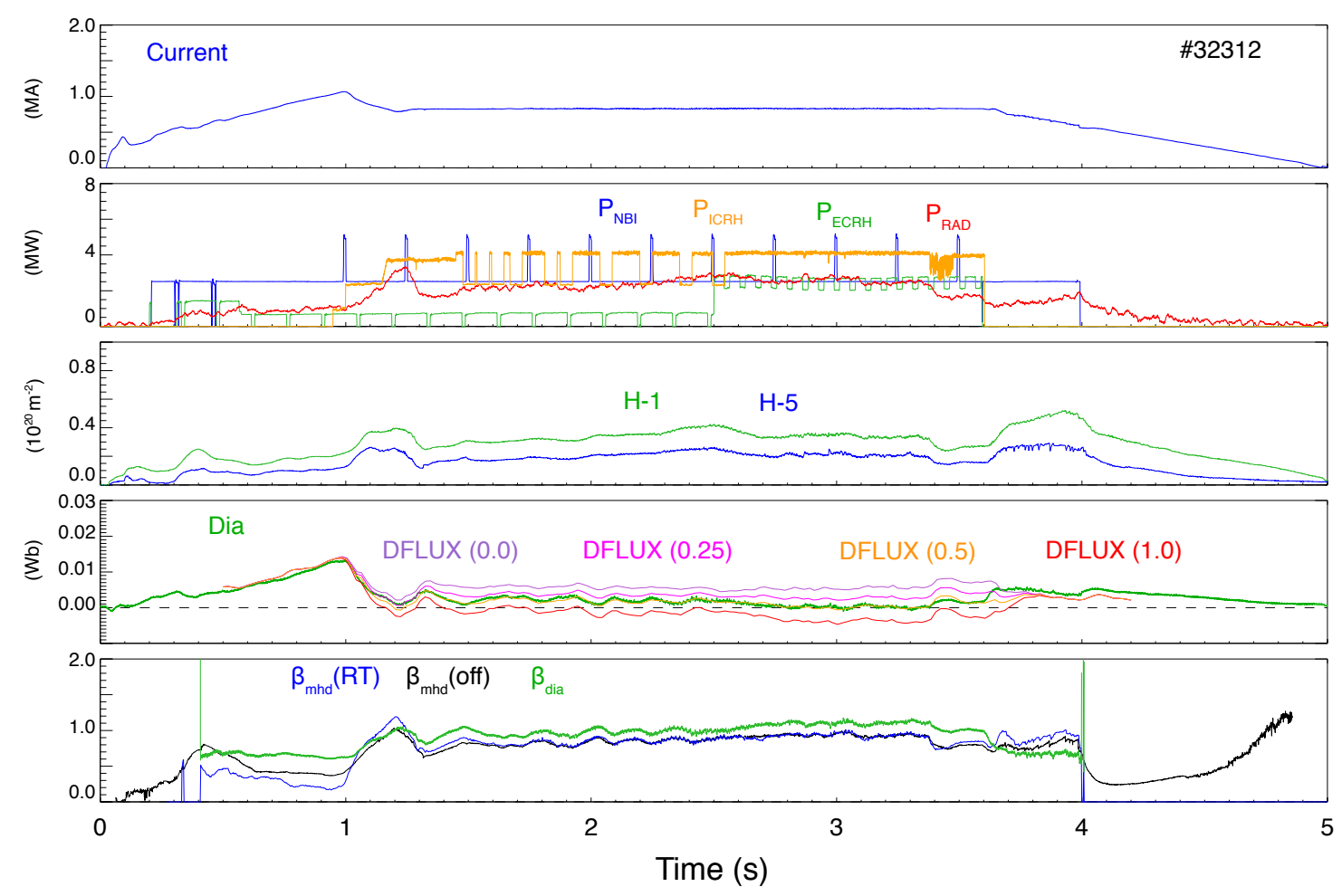

FIG. 7. Comparison of diamagnetic flux with the TRANSP simulation in a discharge with dominant ICRH power. The time evolution of the plasma current and the central and edge electron density line integrals of the DCN laser ( H1 and H5) are shown. The measured diamagnetic flux, Dia (green) and the predicted values from TRANSP (DFLUX) for 4 different fractions of coupled ICRH power are shown. The plasma is anisotropic as the poloidal beta inferred from the measured diamagnetic flux, $\beta_{D I A}$ (green), is greater than the poloidal beta calculated from the real-time and offline magnetic equilibrium ( $\beta_{M H D}(R T)$ (blue) and $\beta_{M H D}($ off $)$ (black) ) during predominant ICRH heating.

predicted by TRANSP was found when it was assumed that only half the nominal generator input power was coupled and that the pressure was anisotropic. 


\section{ACKNOWLEDGMENTS}

This paper is dedicated to J. Gernhardt, the engineer who designed the magnetic probes and diamagnetic loop coils in ASDEX Upgrade. The author would also like to thank the ASDEX Upgrade team members responsible for running and maintaining the tokamak and the plasma diagnostics. The work leading to this article was funded by the European Atomic Energy Community and is subject to the provisions of the European Fusion Development Agreement. This work has been carried out within the framework of the EUROfusion Consortium and has received funding from the Euratom research and training programme 2014-2018 under grant agreement No 633053. The views and opinions expressed herein do not necessarily reflect those of the European Commission.

\section{REFERENCES}

${ }^{1}$ J. Wesson, Tokamaks, 4th ed. (Oxford University Press, Oxford, England, 2011).

${ }^{2}$ J. Gernhardt and F. Schneider, "Design and electronic compensation of a diamagnetic loop and its applications in the ASDEX tokamak," IPP III-84 (Max-Planck-Insitut for Plasmaphysik, 1984).

${ }^{3}$ G. Tonetti, J. P. Christiansen, and L. de Kock, "Measurement of the energy content of the JET tokamak plasma with a diamagnetic loop," Rev. Sci. Instrum. 57, 2087 (1986).

${ }^{4}$ J. Moret, F. Buhlmann, and G. Tonetti, "Fast single loop diamagnetic measurements on the TCV tokamak," Rev. Sci. Instrum. 74, 4634 (2003).

${ }^{5}$ E. J. Strait, "Magnetic diagnostic system of the DIII-D tokamak," Rev. Sci. Instrum. 77 (2006).

${ }^{6} \mathrm{E}$. Joffrin and P. Defrasne, "Differential method for the real time measurement of the diamagnetic and internal inductance in Tore Supra," Rev. Sci. Instrum. 73, 2266 (2002).

${ }^{7}$ J. G. Bak, S. G. Lee, and H. S. Kim, "Diamagnetic loop measurement in Korea Superconducting Tokamak Advanced Research machine," Rev. Sci. Instrum. 82, 063504 (2011).

${ }^{8}$ H. Laqua and F. Schneider, "Improvement of diamagnetic loop measurements in stellarators using digital signal processing techniques," Fusion Eng. Des. 48, 143 (2000).

${ }^{9} \mathrm{~V}$. Shafranov, "Determination of the parameters $\beta_{p}$ and $l_{i}$ in a tokamak for arbitrary shape of plasma pinch cross-section," Plasma Physics 13, 757 (1971). 
${ }^{10}$ E. Lazzaro and P. Mantica, "Experimental identification of tokamak equilibrium using magnetic and diamagnetic signals," Plasma Phys. Contr. Fusion 30, 1735 (1988).

${ }^{11} \mathrm{~J}$. Gernhardt, "Magnetic diagnostic on ASDEX Upgrade with external and internal pick-up coils," Tech. Rep. IPP I-262 (Max-Planck-Insitut for Plasmaphysik, 1992).

${ }^{12}$ V. Shafranov, "Plasma equilibrium in a magnetic field," (Consultant Bureau, New York, 1966) p. 103.

${ }^{13}$ O. Barana, E. Joffrin, A. Murari, F. Sartori, and Contributors to the EFDA-JET Work program, "Real-time determination of confinement parameters in JET," Fusion Eng. Des. 66, 697 (2003).

${ }^{14}$ L. L. Lao, H. E. St. John, H. E. Stambaugh, and W. Pfeiffer, "Separation of $\beta_{p}$ and $l_{i}$ in tokamaks of non-circular cross-section," Nucl. Fusion 25, 1421 (1985).

${ }^{15}$ L. L. Lao, H. E. St. John, H. E. Stambaugh, and W. Pfeiffer, "Reconstruction of current profile parameters and plasma shapes in tokamaks," Nucl. Fusion 25, 1611 (1985).

${ }^{16}$ W. Suttrop, J. C. Fuchs, R. Fischer, L. Giannone, et al., "Mitigation of edge localised modes with magnetic perturbations in ASDEX Upgrade," Fusion Eng. Des. 88, 446 (2013).

${ }^{17}$ R. Harwryluk, "An empirical approach to tokamak transport," in Physics of plasmas close to thermonuclear conditions, Vol. 1, edited by B. Coppi, G. Leotta, D. Pfirsch, R. Pozzoli, and E. Sindoni (Pergamon Press, Oxford, UK, 1980) p. 19.

${ }^{18}$ J. Ongena, M. Evrard, and D. McCune, "Numerical transport codes," Fusion Science Technology 45(2T), 371 (2004).

${ }^{19}$ P.J. McCarthy and ASDEX Upgrade Team, "Identification of edge-localized moments of the current density profile in a tokamak equilibrium from external magnetic measurements," Plasma Phys. Contr. Fusion 54, 015010 (2012).

${ }^{20}$ L. Giannone, M. Reich, M. Maraschek, et al., "A data acquisition system for real-time magnetic equilibrium reconstruction on ASDEX Upgrade and its application to NTM stabilization experiments," Fusion Eng. Des. 88, 3299 (2013).

${ }^{21}$ L. Giannone, R. Fischer, P. McCarthy, et al., "Improvements for real-time magnetic equilibrium reconstruction on ASDEX Upgrade," Fusion Eng. Des. 100, 519 (2015).

${ }^{22}$ A. Kallenbach, M. Bernert, M. Beurskens, L. Casali, M. Dunne, et al., "Partial detachment of high power discharges in ASDEX Upgrade," Nucl. Fusion 55, 053026 (2015).

${ }^{23}$ S. Arshad, R. Budny, J. Cordey, V. Drozdov, S. Gerasimov, D. McDonald, A. Murari, V. Riccardo, I. Voitsekhovich, and JET EFDA contributors, "Consistency of stored energy 
measurements on JET," Tech. Rep. EFDA-JET-CP(05)02-50 (EFDA-JET, 2005). 